\title{
MARÉS ASTRONÔMICAS NA BAÍA DA BABITONGA, SC*.
}

\author{
ELIANE C. TRUCCOLO \& CARLOS A. F. SCHETTINI \\ Laboratório de Oceanografia Física \\ CTTMar - Centro de Ciências Tecnológicas da Terra e do Mar \\ UNIVALI Cx. P. 360 Itajaí SC 88.302.202 \\ Email: nane@cttmar.univali.br \\ * Trabalho financiado pela PETROBRÁs
}

\begin{abstract}
RESUMO
Constantes harmônicas de maré da Praia da Enseada (\# PE) e do porto de São Francisco do Sul (\# PSFS) foram analisadas com o objetivo de se caracterizar a maré astronômica local e conhecer a importância dos efeitos de água rasa na propagação da onda de maré no interior da Baía da Babitonga. O nível do mar na \# PE foi monitorado com marégrafo de pressão por um período de junho a dezembro de 1996. A partir deste monitoramento, um total de 48 constantes harmônicas foram obtidas através da aplicação do método harmônico de maré. Constantes harmônicas para \# PSFS foram obtidas junto à Diretoria de Hidrografia e Navegação, num total de 32 constantes. Baseando-se nestas informações, pode-se caracterizar o regime de maré na Baía da Babitonga como do tipo misto, predominantemente semidiurno com desigualdades de alturas para as preamares e baixamares consecutivas, com Número de Forma de 0,32 para \# PE e 0,29 para \# PSFS. Efeitos não lineares mostraram-se determinantes na caracterização da maré astronômica ocasionando uma amplificação de todas as componentes astronômicas para o interior da baía, caracterizando-a como um estuário hipersíncrono. Este fato é evidenciado pelo aumento das amplitudes em até 7 vezes para algumas constantes e um atraso de fase de 40 minutos. Além do fato do aumento de amplitude das constantes harmônicas, existe uma assimetria na propagação da onda de maré caracterizando a baía pela dominância de enchente de maré. O domínio dos efeitos gerados pela constrição do canal sobre os efeitos de friç̧ão com o fundo foi evidenciado pelo aumento da velocidade dos harmônicos de maré em até $24 \mathrm{~cm} / \mathrm{s}$ para o interior da baía. A baía apresenta um período de oscilação natural de 3,6 horas, cujo período é ressonante com as constantes de espécie quartidiurnas, como a $\mathrm{M}_{4}$ e a $\mathrm{MK}_{4}$, podendo assim ser também um processo importante na amplificação da maré astronômica para o seu interior.
\end{abstract}

Palavras chave: (1) estuário;(2) marés astronômicas; (3) Baía da Babitonga.

\section{ASTRONOMICAL TIDES IN BABITONGA BAY, SC.}

\begin{abstract}
Tidal harmonic constituents for Praia da Enseada (\# PE) and Port of São Francisco do Sul (\# PSFS) were assessed to characterize the local astronomical tide, as well to evaluate the shallow water effects on the tidal propagation to the interior of the Babitonga Bay. The sea level at \# PE were monitored with a pressure tide gauge during June to December, 1996. From this data set, 48 tidal harmonic constituents was determined using the harmonic method for tidal analysis. The 32 harmonic constituents for the \# PSFS were obtained with the Diretoria de Hidrografia e Navegação. Considering these information, the tide at the Babitonga Bay is classified as mixed, with semidiurnal dominance and diurnal inequalities, with Form Number of 0.32 for \# PE and 0,29 for \# PSFS. Nonlinear effects results in the amplification of the astronomical constituents towards the interior of the bay, showing hypersynchronous behavior. Some harmonic constituents increased about 2 to 7 times, and all presented around of 40 minute phase shift. Despite of the astronomical constituents amplification, there are also
\end{abstract}


an asymmetry on the wave propagation, with flood dominate in bay. The role of the channel constriction effects over the bottom friction was clearly observed by the harmonic constituents speed increase towards the bay. The Babitonga Bay presents a natural oscillation period about 3,6 hours resonance with some of shallow water components. This effect can be a significant processes in the increasing of the astronomical components.

Keywords: (1) estuary; (2) astronomical tide; (3) Babitonga Bay.

\section{INTRODUÇÃO}

O nível do mar costeiro está continuamente oscilando em resposta às forçantes astronômicas, atmosféricas e oceanográficas, em diferentes escalas de tempo e espaço (Gill, 1982; Pugh, 1987). O espectro temporal destas flutuações varia de poucas horas até vários dias. Tais variações ocorrem principalmente em resposta à forçantes (1) de origem astronômica, de natureza determinística, e (2) devido à ação do vento e pressão atmosférica sobre o oceano, de difícil previsão devido à natureza aleatória do fenômeno atmosférico.

A maré astronômica é o movimento periódico de subida e descida do nível do mar que obedece à força do campo gravitacional no sistema Terra-Sol-Lua (Pugh, 1987). A onda de maré é uma onda longa e suas características são modificadas quando avança do oceano profundo para as plataformas continentais. Distorções da onda são notadas mais facilmente na sua propagação em regiões de pouca profundidade e com restrições laterais como em baías e estuários.

A progressão da onda de maré em águas rasas é influenciada por efeitos não lineares que contribuem na distorção e dissipação de energia. Entre os efeitos estão: (1) a influência da fricção do fundo e constrição lateral do canal; (2) a configuração da costa que impõem variações na direção de fluxos de marés, e (3) a profundidade da coluna de água, que agora é comparável em escala com a amplitude da maré (Pugh, 1987; Dyer, 1997).

O nível do mar também pode oscilar devido à efeitos induzidos por processos meteorológicos. Estas oscilações, quando simultâneas às marés de sizígia, podem acarretar níveis extraordinários muito acima dos níveis possíveis devido somente à maré astronômica. Assim sendo, o conhecimento da importância relativa da maré astronômica e seu comportamento em ambientes aquáticos costeiros é uma informação essencial no seu gerenciamento apropriado.

A Baía da Babitonga situa-se no litoral norte de Santa Catarina, sendo o maior ambiente estuarino de Santa Catarina. A baía banha as cidades de Joinville e São Francisco do Sul, apresentando uma extensa área de manguezal, principalmente nas suas porções mais interiores. O objetivo deste artigo é o de avaliar o comportamento da maré astronômica e a progressão da onda de maré na Baía da Babitonga.

\section{DADOS UTILIZADOS}

Informações sobre o regime de maré foram obtidos para duas localidades: na praia da Enseada (26¹3'09'S, 48²9'05"W) e no porto de São Francisco do Sul (26¹4'05"S, $48^{\circ} 38^{\prime} 02^{\prime \prime W}$ ). A primeira estação está localizada na costa e a segunda distante $14 \mathrm{~km}$ à montante da barra da baía (Figura 1). Os dados consistem de constantes harmônicas de maré para os dois locais. As constantes harmônicas para o porto de São Francisco do Sul foram obtidas junto à Diretoria de Hidrografia e Navegação (DHN). As constantes harmônicas para a praia da Enseada foram obtidas aplicando-se o método harmônico descrito em Franco (1988), através do programa do mesmo autor Pacmaré (Previsão e Análise de Maré), sobre uma série de seis 
meses de monitoramento com freqüência amostral de 0,5 hora obtida com um marégrafo de pressão Aanderaa ${ }^{\mathrm{TM}}$.

Um total de 48 constantes estatisticamente significativas com nível de confiança de $95 \%$ foram obtidas para a praia da Enseada, sendo 14 constantes diurnas e semidiurnas e 34 constantes de água rasa. Das 32 constantes do porto de São Francisco do Sul (PSFS), 20 são diurnas e semidiurnas e 12 são constantes de água rasa. Na Tabela 1 são mostradas as 20 constantes harmônicas comuns para ambas estações. Cada constituinte é descrito pelo seu período, amplitude e fase. Os constituintes resultantes para \# PE apresentam períodos de até 27 horas, sendo que nenhum constitu- inte de longo período foi obtido ao nível de 95\% de significância estatística.

\section{REGIME E PROGRESSÃO DA MARÉ}

O regime da maré astronômica para os dois locais podem ser descritos a partir dos valores de amplitude dos constituintes harmônicos (Schureman, 1941; Palmer et al., 1980). Um resumo destas características são listadas na Tabela 2. A altura média de maré na estação \# PSFS é $85 \mathrm{~cm}$, sendo $21 \%$ maior do que a altura média na estação \# PE, de 70 $\mathrm{cm}$. Os valores máximos de altura durante os períodos de sizígia são 128 e $106 \mathrm{~cm}$, e os valores de altura mínimos durante os períodos de quadratura são 27 e $21 \mathrm{~cm}$, respecti-
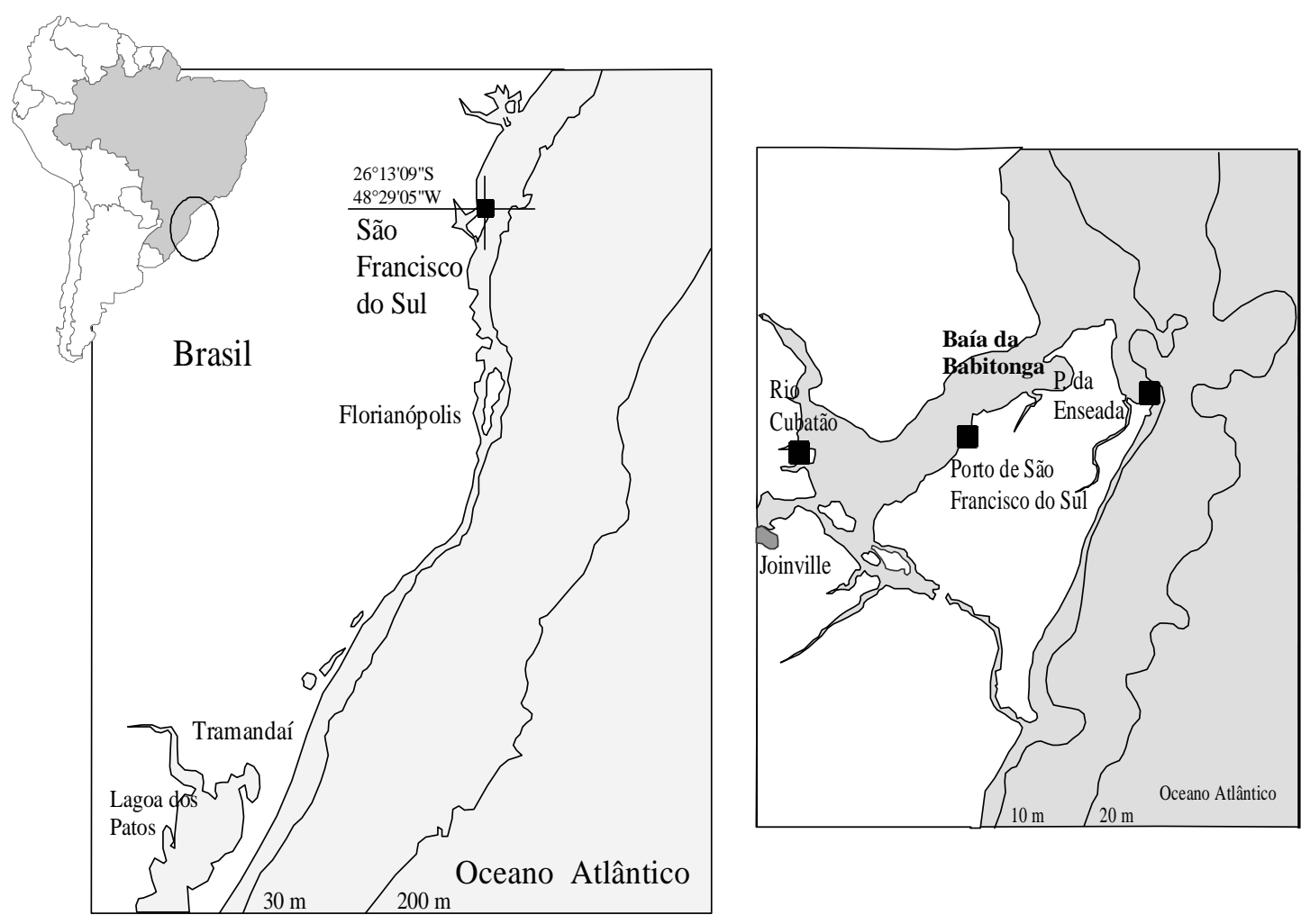

Figura 1: Localização das estações maregráficas da Praia da Enseada, do Porto de São Francisco do Sul e do Rio Cubatão. 
TRUCCOLO \& SCHETTINI: Marés astronômicas na Baía da Babitonga.

Tabela 1: Constituintes harmônicas comuns entre a Praia da Enseada e o Porto de São Francisco do Sul estatisticamente significativas.

\begin{tabular}{|c|c|c|c|c|c|}
\hline \multirow[t]{2}{*}{ Espécie } & \multirow{2}{*}{$\begin{array}{l}\text { Período } \\
\text { (horas) }\end{array}$} & \multicolumn{2}{|c|}{ \# PE } & \multicolumn{2}{|c|}{ \# PSFS } \\
\hline & & $\begin{array}{l}\text { Amplitude } \\
\text { (cm) }\end{array}$ & Fase (graus) & $\begin{array}{l}\text { Amplitude } \\
\text { (cm) }\end{array}$ & Fase (graus) \\
\hline \multicolumn{6}{|l|}{ Diurnas } \\
\hline Q1 & 26,87 & 3,2 & 47,5 & 3,4 & 57,7 \\
\hline O1 & 25,82 & 10,8 & 73,6 & 11,4 & 73,0 \\
\hline P1 & 24,07 & 2,4 & 99,7 & 2,4 & 126,7 \\
\hline K1 & 23,93 & 6,0 & 141,5 & 6,8 & 132,0 \\
\hline \multicolumn{6}{|c|}{ Semi-diurnas } \\
\hline $2 \mathrm{~N} 2$ & 12,91 & 1,8 & 129,6 & 2,4 & 138,2 \\
\hline N2 & 12,66 & 4,8 & 133,9 & 6,9 & 150,9 \\
\hline M2 & 12,42 & 31,6 & 64,5 & 38,7 & 76,9 \\
\hline L2 & 12,19 & 1,9 & 70,7 & 2,5 & 84,5 \\
\hline S2 & 12,00 & 21,2 & 65,7 & 25,0 & 75,6 \\
\hline K2 & 11,97 & 7,1 & 60,9 & 8,9 & 62,7 \\
\hline \multicolumn{6}{|c|}{ Água Rasa } \\
\hline MKS2 & 12,38 & 2,1 & 108,9 & 11,6 & 71,5 \\
\hline MO3 & 8,39 & 4,5 & 332,1 & 7,6 & 15,9 \\
\hline SO3 & 8,29 & 3,0 & 92,0 & 3,4 & 212,1 \\
\hline M3 & 8,28 & 6,9 & 180,1 & 11,2 & 217,3 \\
\hline MK3 & 8,18 & 2,7 & 78,1 & 3,7 & 113,5 \\
\hline SK3 & 7,99 & 2,6 & 263,7 & 2,9 & 232,5 \\
\hline MN4 & 6,27 & 4,7 & 87,7 & 6,9 & 149,6 \\
\hline M4 & 6,21 & 10,7 & 132,9 & 17,3 & 196,8 \\
\hline MS4 & 6,11 & 5,0 & 215,7 & 8,0 & 291,8 \\
\hline MK4 & 6,10 & 1,8 & 251,9 & 13,3 & 292,6 \\
\hline Soma & & 134,8 & & 194,3 & \\
\hline
\end{tabular}

vamente para \# PSFS e \# PE. A variação das constantes de água rasa apresentaram uma acentuada variação entre as duas estações, alcançando valores maiores que as constantes diurnas. Segundo o esquema de classificação de Hayes (1975), ambas as estações podem ser caracterizadas por um regime de micro-marés, com a máxima altura de maré inferior à $2 \mathrm{~m}$.
A importância relativa das constantes diurnas e semidiurnas de maré pode ser avaliada através do Número de Forma (Pugh, 1987), definido pela razão da soma das amplitudes dos principais constituintes diurnos pela soma das amplitudes das principais constantes semidiurnas $\left(A_{K 1}+A_{O_{1}}\right) /\left(A_{M 2}+A_{S 2}\right)$. Os Números de Forma para \# PE e \# PSFS são 0,32 e 0,29 , respectivamente, o que repre- 
NOTAS TÉC. FACIMAR, 3: 57-66, 1999.

Tabela 2: Parâmetros descritores da maré astronômica nas duas estações. O número de forma é adimensional, e os demais valores estão em centímetros.

\begin{tabular}{cccc}
\hline Parâmetro & Fórmula & \# PE & \# PSFS \\
\hline Número de forma & $\left(\mathrm{K}_{1}+\mathrm{O}_{1}\right) /\left(\mathrm{M}_{2}+\mathrm{S}_{2}\right)$ & 0,32 & 0,29 \\
Altura média & $2,2\left(\mathrm{M}_{2}\right)$ & 70,0 & 85,0 \\
Altura de sizígia & $2,0\left(\mathrm{M}_{2}+\mathrm{S}_{2}\right)$ & 106,0 & 128,0 \\
Altura de quadratura & $2,0\left(\mathrm{M}_{2}-\mathrm{S}_{2}\right)$ & 21,0 & 27,0 \\
\hline
\end{tabular}

Tabela 3: Amplitude e porcentagem das constantes harmônicas diurnas, semidiurnas e de água rasa para a Praia da Enseada (\# PE) e Porto de São Francisco do Sul Sul (\# PSFS).

\begin{tabular}{ccccc}
\hline Espécie & $\begin{array}{c}\text { Somatório \# PE } \\
(\mathrm{cm})\end{array}$ & $\begin{array}{c}\text { Percentural \# PE } \\
(\%)\end{array}$ & $\begin{array}{c}\text { Somatório \# PSFS } \\
(\mathrm{cm})\end{array}$ & $\begin{array}{c}\text { Percentural \# PSFS } \\
(\%)\end{array}$ \\
\hline Diurnas & 22,0 & 16,4 & 24,0 & 12,1 \\
Semi-diurnas & 70,0 & 51,4 & 87,0 & 44,2 \\
de Água rasa & 44,0 & 32,2 & 86,0 & 43,7 \\
\hline
\end{tabular}

senta um regime de maré do tipo misto com predominância semidiurna, com desigualdades de alturas para as preamares e baixamares consecutivas.

As constantes de espécies diurnas representam aproximadamente $16 \%$ e $12 \%$ do somatório das amplitudes das constantes para \# PE e \# PSFS, respectivamente. As constantes de espécies semi-diurnas são responsáveis por $51,4 \%$ da energia da maré em \# $\mathrm{PE}$, sendo os $32,2 \%$ restantes devido à constantes de água rasa. Para \# PSFS as constantes semidiurnas apresentam importância equivalente às constantes de água rasa, representando $44,2 \%$ e $43,7 \%$ da energia da maré, respectivamente (Tabela 3 ).

Em ambas as estações as constantes principal lunar $\mathrm{M}_{2}$ e principal solar $\mathrm{S}_{2}$ são as mais energéticas, apresentando a primeira 31,6 e $38,7 \mathrm{~cm}$ e a segunda 21,2 e 25,0 para \# PE e \# PSFS, respectivamente. Isto representa um aumento na amplitude de $22,5 \%$ para a $M_{2}$ e $17,9 \%$ para a $S_{2}$, havendo um atraso de fase em torno de 10 graus $(40 \mathrm{mi}-$ nutos) para ambas as constantes. As cons- tantes que apresentaram maiores variações nas suas amplitudes entre \# PE e \# PSFS, foram: $\mathrm{MK}_{4}$, com um aumento de 7,5 vezes; $\mathrm{MKS}_{2}$ com aumento de 5,5 vezes; $\mathrm{M}_{3}$ e $\mathrm{M}_{4}$ com aumento de 1,6 vezes. Todas estas constantes são espécies de água rasa.

O efeito de amplificação da energia de maré a partir da estação \# PE até a estação \# PSFS é mostrado para um período de sete dias de sizígia na Figura 2, e para um período de 7 dias de quadratura na Figura 3 . Em ambos os casos pode-se observar variações sinópticas da ordem de $50 \mathrm{~cm}$ e a existência de um atraso de fase.

Além da amplificação da energia de maré a partir da estação \# PE para a estação \# PSFS, ocorre também distorções da onda neste trecho decorrente do seu avanço para um ambiente restrito. Segundo Speer e Aubrey (1985), a maior parte da assimetria da curva de maré astronômica é resultado do balanço entre a topografia do estuário e os efeitos de fricção e estreitamento do canal. A distorção da onda causa a dominância de enchente ou de vazante, podendo ser avalia- 
da pela análise das fases da constante principal lunar $\mathrm{M}_{2}$ e de uma de suas constantes filhas geradas durante a progressão da maré em águas rasas, a $\mathrm{M}_{4}$, pela relação $\left(2 q_{\mathrm{M} 2}\right.$ $\left.\mathrm{q}_{\mathrm{M} 4}\right)$. Para um sistema ser caracterizados como dominado por enchente, este deve apresentar uma elevação de fase entre $0^{\circ}$ e $180^{\circ}$, e em caso de valores entre $180^{\circ}$ e $360^{\circ}$, o sistema é caracterizado por domínio de vazante. Esta relação é $4^{\circ}$ para \# PE e $43^{\circ}$ para \# PSFS. Valores próximos à $0^{\circ} / 360^{\circ}$ ou $180^{\circ}$ implicam em um regime quasi-simetrico, sendo o caso da maré na Praia da Enseada, em mar aberto. Entretanto, na estação \# PSFS a relação de simetria mostra uma forte tendência para domínio de marés de enchente.

A Figura 4 mostra claramente a amplificação e o atraso que ocorre na progressão da maré para o interior da baía. A linha tracejada representa a variação do nível do mar observada na barra do Rio Cubatão, que deságua no Rio Palmital que é o braço norte da baía. Observa-se a ocorrência da baixa mar em \# PE antecedendo à de \# PSFS, que por sua vez antecede à observada na barra do Rio Cubatão. Neste mesmo período, a altura alcançada em \# PE foi de $1,30 \mathrm{~m}$, aumentando para $1,87 \mathrm{~m}$ em \# PSFS, e decaindo para 1,70 na barra do Rio Cubatão.

Este comportamento sugere que a Baía da Babitonga é um estuário do tipo hipersíncrono, onde o aumento das amplitudes está relacionado com a magnitude relativa entre os efeitos de fricção com o fundo e o estreitamento lateral do canal de acesso (Nichols \& Biggs, 1985). É possível observar
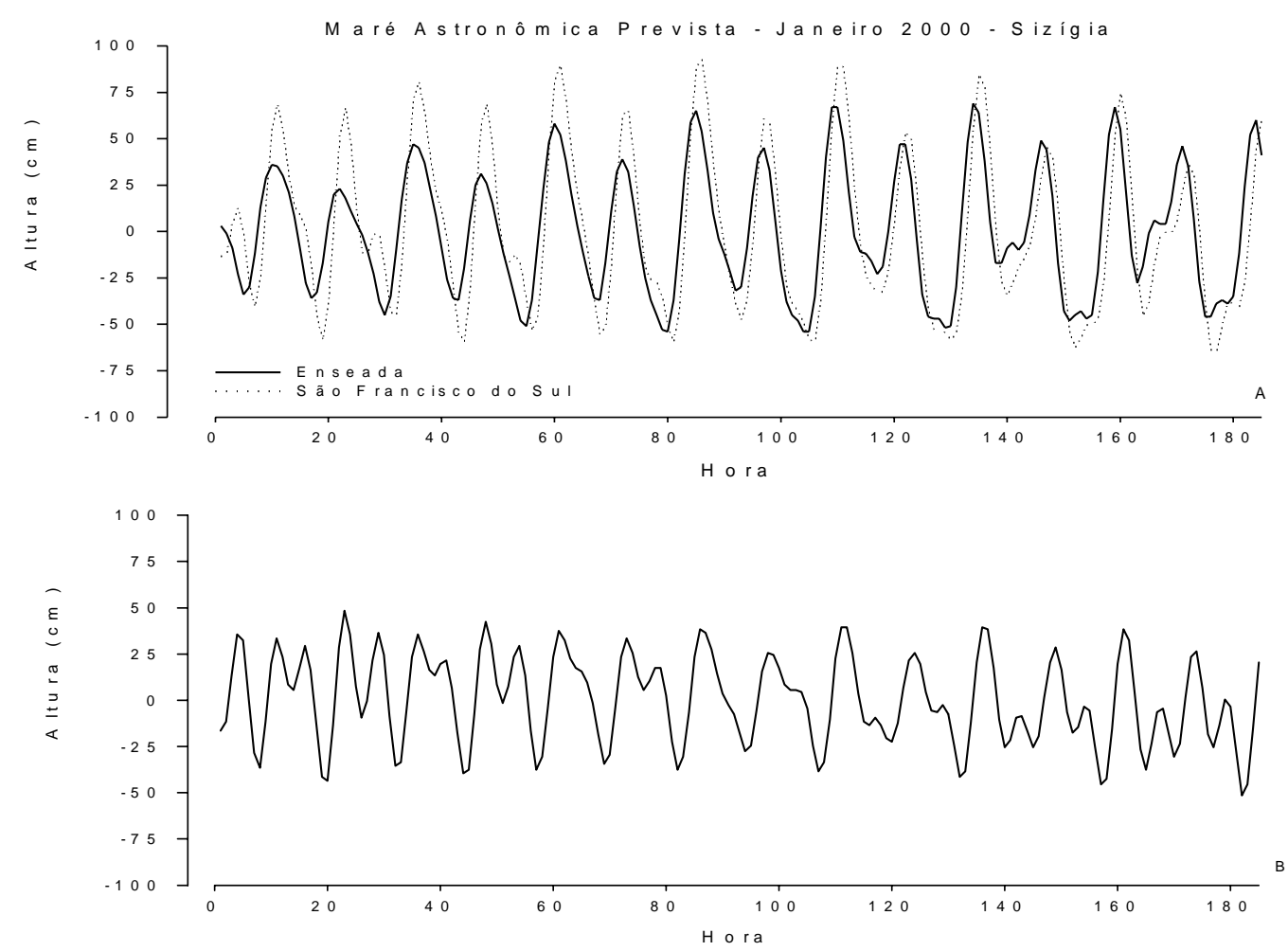

Figura 2: (a) maré astronômica prevista para o Porto de São Francisco do Sul (linha fina) e a Praia da Enseada (linha grossa) para Janeiro de 2000, durante um período de sizígia; (b) diferença de nível entre as estações. 
o aumento das amplitudes de constituintes mareais ao longo de um estuário se forem consideradas relações entre a fricção com o fundo e a convergência lateral do canal. Quando os efeitos de constrição do canal são dominantes e a fricção tem um papel menor, a constrição causa uma compressão da onda lateralmente e a conservação de energia requer que as velocidades e amplitudes da onda de maré aumentem (Dyer, 1997).

A variação das amplitudes e fases dos constituintes de maré observados ao longo do estuário afetarão as velocidades de correntes de maré. É possível caracterizar a velocidade média de propagação da onda de maré entre a estação PE e estação PSFS através de (Franco, 1988):

$$
\tilde{v}=3[g(\varsigma+\bar{p})]^{1 / 2}-2(g \bar{p})^{1 / 2}
$$

onde $\tilde{v}$ é a velocidade da onda, $g$ é aceleração da gravidade $\left(9,81 \mathrm{~m}^{-\mathrm{s}^{-2}}\right), \bar{p}$ é a profundidade média ( $5 \mathrm{~m})$, zé a amplitude dos constituintes. O tempo requerido para que esta onda chegue à uma seção é obtido por:

$$
\Delta t=\Delta x / \tilde{v}
$$

onde $\mathrm{D} X$ é a distância entre as duas estações.

Ao considerar as velocidades médias das ondas de maré e o tempo gasto ao pro-
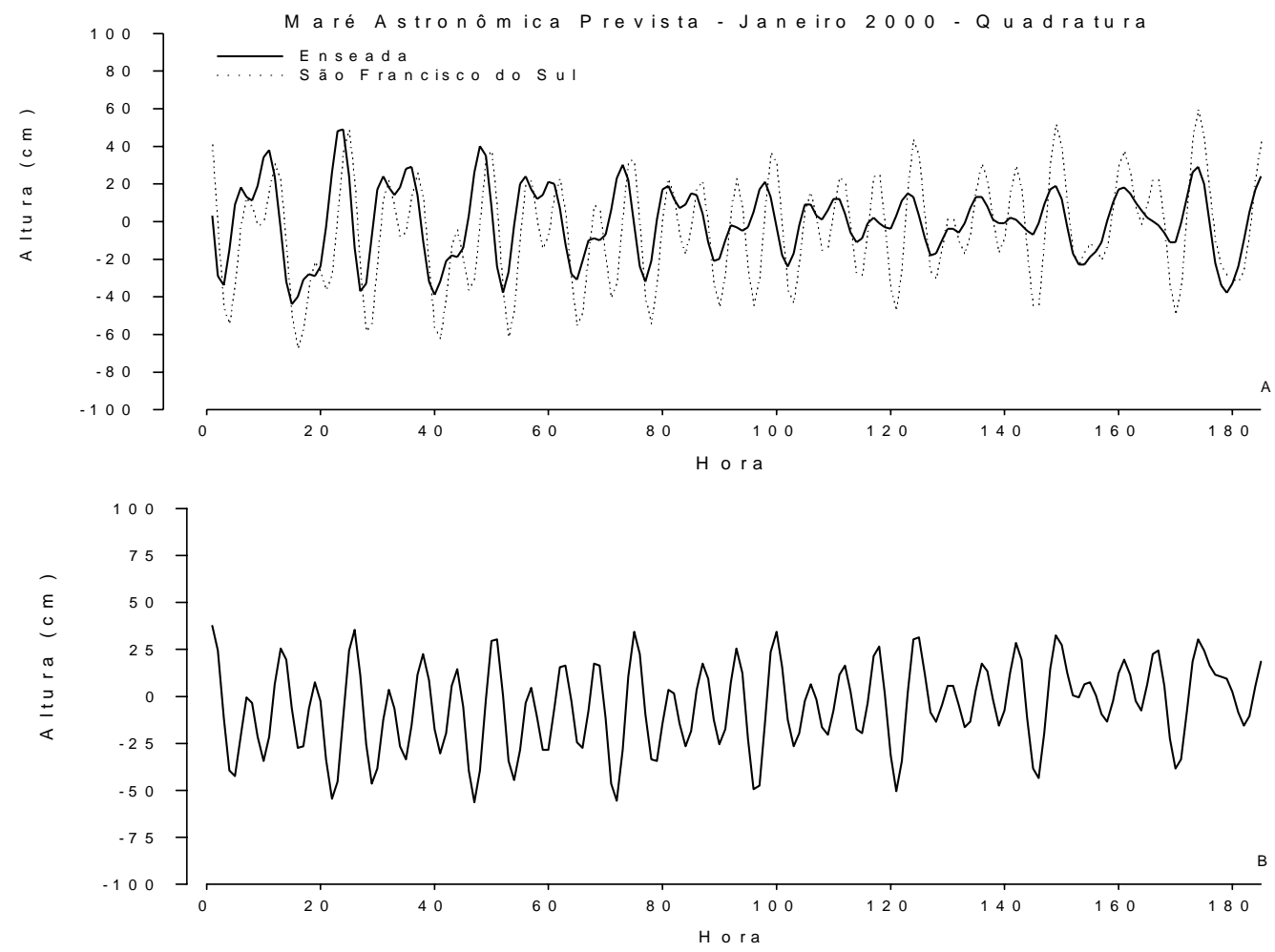

Figura 3: (a) maré astronômica prevista para o Porto de São Francisco do Sul (linha fina) e a Praia da Enseada (linha grossa) para Janeiro de 2000, durante um período de quadratura; (b) diferença de nível entre as estações. 
pagarem-se de \# PE até \# PSFS (Tabela 4), pode-se observar que ocorrem diferenças entre as velocidades dos principais harmônicos entre as duas estações. Levando-se em consideração a profundidade média da baía de $5 \mathrm{~m}$, foi observado aumentos na velocidade em até $24 \mathrm{~cm} / \mathrm{s}$ para as constituintes de água rasa, e para a semidiurna $\mathrm{M}_{2}$ uma diferença de velocidade de $14 \mathrm{~cm} / \mathrm{s}$. A diferença das amplitudes médias foi de até $12 \mathrm{~cm}$ entre a estação costeira e interior. $O$ tempo médio decorrido de 33 minutos foi observado para todas as componentes para o percurso entre a \# PE e \# PSFS.

\section{PERÍODO DE OSCILAÇÃO NATURAL}

O aumento da amplitude da maré entre \# PE e \# PSFS pode ser gerado também pela sobreposição da maré astronômica com ondas geradas pela oscilação natural do corpo de água. O período de oscilação natural de um corpo de água é o tempo dado para uma onda se propagar até o outro lado do sistema, sofrer reflexão e retornar. Caso o período de oscilação natural seja semelhante ou múltiplo de algum constituinte harmônico, poderá ocorrer à amplificação em determinadas freqüências. O período de oscilação natural de um corpo d'água pode ser calculado pela Fórmula de Merian (Pugh, 1987):

$$
P O N=\frac{4 e}{(g p)^{1 / 2}}
$$

onde e é a extensão do sistema ( $₫ 22,5$ $\mathrm{km})$. O período de oscilação natural para a Baía da Babitonga é em torno de 3,6 horas.

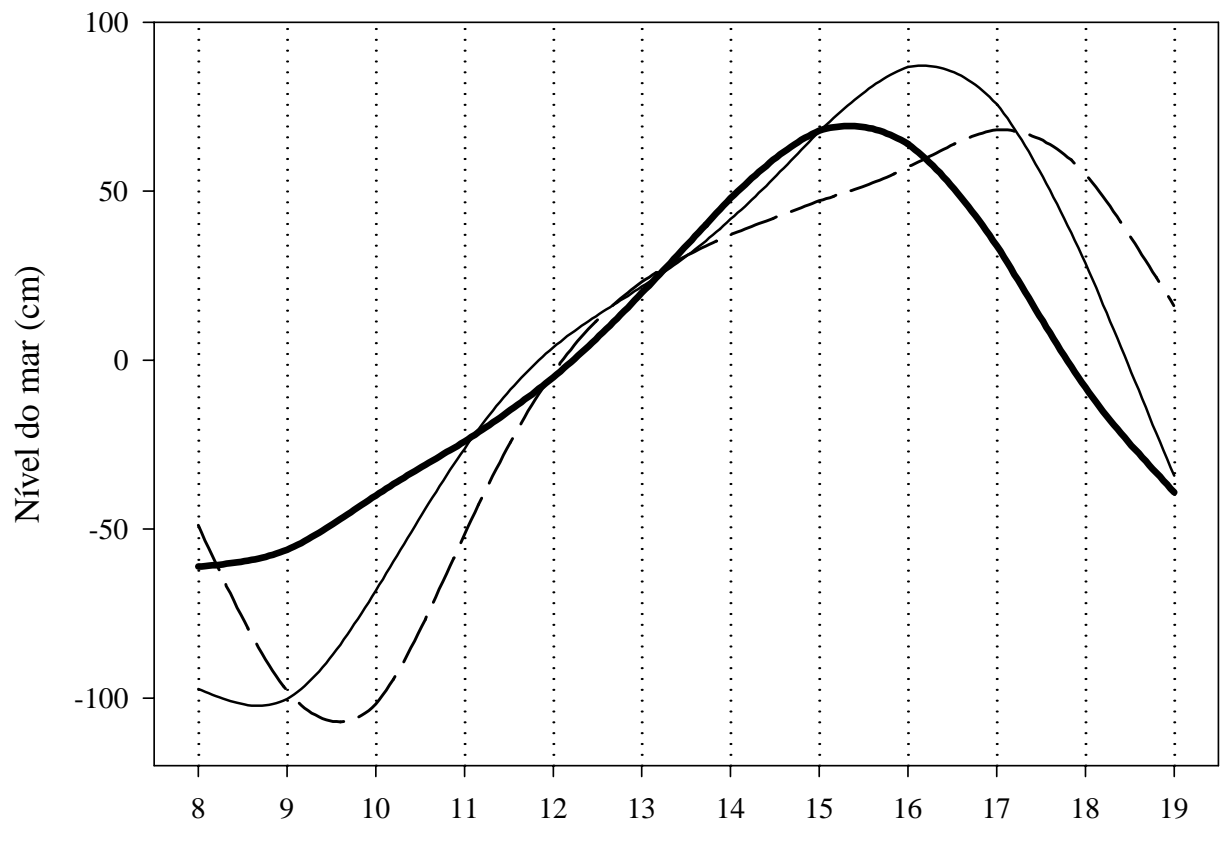

Horas, 2 de maio de 1999

Figura 4: Variação do nível do mar ao longo da Baía da Babitonga, no dia 2 de maio de 1999. Praia de Enseada: linha contínua em negrito; Porto de São Francisco do Sul: linha contínua; Barra do Rio Cubatão: linha tracejada. Nível do mar observado para barra do Rio Cubatão, e predita para os outros pontos. 
Foi observado uma relação entre o período natural e as constituintes astronômicas cujas freqüências são múltiplas deste período, incluindo a $\mathrm{M}_{4}$ e a $\mathrm{MK}_{4}$. Neste caso parece ocorrer o fenômeno de ressonância, onde amplificação pode ser gerada se um componente tiver uma freqüência múltipla de outra freqüência. Portanto, sistemas que são forçados por oscilações próximas ao seu período natural respondem aumentando a amplitude e apresentam um comportamento ressonante, porém, se o comprimento da baía for somente uma fração do comprimento da onda de maré então a amplificação é pequena, e outros processos estarão atuando (Pugh, 1987).

Tabela 4: Característica da progressão da onda de maré entre a Praia de Enseada (\# PE) e o Porto de São Francisco do Sul (\# PSFS) para as principais constantes harmônicas.DT: tempo para percorrer a distâncias entre os dois pontos; C: celeridade da onda; DA: variação da amplitude; DC: variação da celeridade.

\begin{tabular}{|c|c|c|c|c|c|}
\hline Espécie & $\Delta \mathrm{T}(\mathrm{min})$ & $\mathrm{C}_{\mathrm{PE}}\left(\mathrm{m} \cdot \mathrm{s}^{-1}\right)$ & $\mathrm{C}_{\mathrm{PSFS}}\left(\mathrm{m} \cdot \mathrm{s}^{-1}\right)$ & $\Delta \mathrm{A}(\mathrm{cm})$ & $\Delta \mathrm{C}\left(\mathrm{m} \cdot \mathrm{s}^{-1}\right)$ \\
\hline \multicolumn{6}{|l|}{ Diurnas } \\
\hline Q1 & 33 & 7,07 & 7,07 & 0,20 & 0,00 \\
\hline O1 & 32 & 7,23 & 7,24 & 0,60 & 0,01 \\
\hline P1 & 33 & 7,05 & 7,05 & $-0,10$ & 0,00 \\
\hline K1 & 33 & 7,13 & 7,15 & 0,80 & 0,02 \\
\hline \multicolumn{6}{|c|}{ Semi-diurnas } \\
\hline $2 \mathrm{~N} 2$ & 33 & 7,04 & 7,05 & 0,60 & 0,01 \\
\hline N2 & 33 & 7,10 & 7,15 & 2,10 & 0,04 \\
\hline M2 & 30 & 7,66 & 7,80 & 7,10 & 0,14 \\
\hline L2 & 33 & 7,04 & 7,06 & 0,60 & 0,01 \\
\hline $\mathrm{S} 2$ & 31 & 7,44 & 7,52 & 3,80 & 0,08 \\
\hline $\mathrm{K} 2$ & 33 & 7,15 & 7,19 & 1,80 & 0,04 \\
\hline \multicolumn{6}{|l|}{ Água rasa } \\
\hline MKS2 & 33 & 7,05 & 7,25 & 9,50 & 0,20 \\
\hline MO3 & 33 & 7,10 & 7,16 & 3,10 & 0,06 \\
\hline SO3 & 33 & 7,07 & 7,07 & 0,40 & 0,01 \\
\hline M3 & 33 & 7,15 & 7,24 & 4,30 & 0,09 \\
\hline MK3 & 33 & 7,06 & 7,08 & 1,00 & 0,02 \\
\hline SK3 & 33 & 7,06 & 7,06 & 0,30 & 0,01 \\
\hline MN4 & 33 & 7,10 & 7,15 & 2,20 & 0,05 \\
\hline M4 & 32 & 7,23 & 7,36 & 6,60 & 0,14 \\
\hline MS4 & 33 & 7,11 & 7,17 & 3,00 & 0,06 \\
\hline MK4 & 33 & 7,04 & 7,28 & 11,50 & 0,24 \\
\hline
\end{tabular}




\section{CONCLUSÕES}

O regime de maré na Baía da Babitonga é do tipo misto, predominantemente semidiurno com desigualdades de alturas para as preamares e baixa-mares consecutivas. A amplitude média de maré para a Praia da Enseada (\# PE) e Porto de São Francisco do Sul (\# PSFS) são 70 e $85 \mathrm{~cm}$, respectivamente, com valores máximos de 106 e 128 $\mathrm{cm}$, na mesma ordem.

A importância relativa entre as constantes semidiurnas e as constantes de água rasa para \# PE e \# PSFS mostram que efeitos não lineares neste trecho são determinantes na caracterização da maré astronômica. Este fato é evidenciado pelo aumento das amplitudes em até 7 vezes para algumas constantes.

A Baía da Babitonga é caracterizada pela dominância de enchente de maré. Esta assimetria na propagação da onda de maré depende do balanço entre os efeitos de fricção e estreitamento do canal. A baía pode ser caracterizada como um estuário do tipo hipersíncrono, onde os efeitos de constrição do canal parecem ser dominantes sobre os efeitos friccionais. As variações de amplitude e fase da maré devido ao estreitamento afeta as velocidades de corrente de maré ao longo do estuário. Este comportamento é comprovado pelos aumentos na velocidade em até $24 \mathrm{~cm} / \mathrm{s}$ de algumas constantes harmônicas e um atraso médio de fase entre \# PE e \# PSFS de 33 minutos.

A baía apresenta um período de oscilação natural de 3,6 horas, cujo período é ressonante com as constantes de espécie quartidiurnas, como a $\mathrm{M}_{4}$ e a $\mathrm{MK}_{4}$, podendo assim ser também um processo importante na amplificação da maré astronômica para o seu interior.

\section{REFERÊNCIAS BIBLIOGRÁFICAS}

Dyer, K. 1997. Estuaries, A Physical Introduction. $2^{\circ}$ Ed. New York, John Wiley and Sons, 195pp.

Franco, A. S. 1988. Tides - Fundamentals, Analysis and Prediction. $2^{\circ}$ Ed., Fundação Centro Tecnológico de Hidráulica (FCTH), São Paulo, Brasil. 249pp.

Gill, A E. 1982. Atmosphere-Ocean Dynamics. International Geophysics Series, Vol. 30. London, Academic Press, 662pp.

Hayes, M.O. 1975. Morphology of sand accumulation in estuaries: an introduction to the symposium. In: Cronin, L.E. (Ed.). Estuarine Research, Vol. II, New York, Academic Press, pp. 3-22.

Nichols, M.M. \& Biggs, R.B. 1985. Estuaries. In: Davis Jr., R.A. (Ed.). Coastal sedimentary environments. New York, John Wiley and Sons, 465pp.

Palmer, M.A., Kjerfve, B., and Schwing, F.B. 1980. Tidal analysis and prediction in a South Carolina estuary. Contributions in Marine Science, 23:17-23.

Pugh, D.T. 1987. Tides, surges and mean sea level, a handbook for engineers and scientists. New York, John Wiley and Sons, 472pp.

Schureman, P. 1941. Manual of harmonic analysis and prediction of tides. Coast and Geodetic Survey Special Publication 98. United States Government Printing Office. Washington, DC. 313pp.

Speer, P. E. e Aubrey, D. G. 1985. A study of non-linear tidal propagation in shallow inlet-estuarine systems. Part II: Theory. Est. Coast. Shelf Sci. 21, 207-224 